\title{
Accuracy of kinetic perimetry assessment with the Humphrey 850; an exploratory comparative study
}

\author{
Fiona J. Rowe ${ }^{1} \cdot$ Lauren R. Hepworth $\mathbb{1}^{1} \cdot$ Kerry L. Hanna ${ }^{1} \cdot$ Meera Mistry ${ }^{1}$ Carmel P. Noonan ${ }^{2}$
}

Received: 20 July 2018 / Revised: 7 May 2019 / Accepted: 17 June 2019 / Published online: 22 July 2019

(c) The Author(s), under exclusive licence to The Royal College of Ophthalmologists 2019

\begin{abstract}
Aim To compare kinetic perimetry on the Humphrey 850 and Octopus 900 perimeters for assessment of visual fields, uniocular rotations and fields of binocular single vision.

Methods Prospective cross section study comparing Humphrey 850 kinetic perimetry to kinetic perimetry using the Octopus 900. Results were compared for both perimeters for the measurement of visual field boundaries, uniocular rotations and fields of binocular single vision in subjects with normal visual function, with comparisons of mean vector extremity values and duration of testing. A visual field boundary overlay was used to assess detection potential of Humphrey 850 kinetic perimetry using I4e and I2e targets in results of known abnormal visual fields.

Results Fifteen subjects (30 eyes) with normal parameters of visual function underwent dual perimetry assessment. Mean visual field boundaries and ocular rotation extremity values were similar for Humphrey and Octopus kinetic perimetry along horizontal meridians. Measurements for Humphrey perimetry were significantly smaller for superior and inferior visual field and rotations with ceiling effects at approximately 40 and 50 degrees, respectively. Use of visual field boundary overlays for 140 patient results showed high detection of the known abnormal visual field results by the Humphrey 850 perimeter $(91.4 \%$ with I4e target; 95\% with I2e target) but with notable exceptions for peripheral superior visual field defects.

Conclusions The Humphrey perimeter's aspheric bowl introduces a ceiling effect for measurements in the superior and inferior visual field at approximately 40 and 50 degrees respectively. This results in potential diagnostic accuracy issues when measuring uniocular rotations, fields of binocular single and visual field boundaries in conditions that specifically impair superior and/or inferior ocular motility (e.g., thyroid eye disease) or visual fields (e.g., chiasmal compression).
\end{abstract}

\section{Introduction}

Perimetry is a cornerstone assessment for many ocular and neurological conditions. Quantitative kinetic perimetry has been a key assessment since the 1940's with the introduction of the Goldmann perimeter ${ }^{\mathrm{TM}}$ (Haag Streit International, Koeniz, Switzerland). Static perimetry was introduced in the 1970's (e.g., Octopus 101 ${ }^{\mathrm{TM}}$, Humphrey field analyzer ${ }^{\mathrm{TM}}$ ) and quickly became a popular assessment choice with advantages over kinetic perimetry in relation to

Fiona J. Rowe

rowef@liverpool.ac.uk

1 Department of Health Services Research, University of Liverpool, Liverpool L69 3GB, UK

2 Department of Ophthalmology, Aintree University Hospital NHS Foundation Trust, Liverpool L7 9AL, UK earlier detection of visual field loss through static thresholding strategies, repeatability of test programmes and reduced inter-examiner bias [1-3].

Kinetic perimetry maintained advantages over static perimetry in the assessment of severe visual field loss, in patients with poor central vision and fixation, in the assessment of children and individuals with reduced cognitive function and in the assessment of ocular rotations and field of binocular single vision [4-10]. Goldmann perimetry was often the kinetic perimeter of choice until its replacement with the Octopus $900^{\mathrm{TM}}$ (Haag Streit International, Koeniz, Switzerland) in 2007. Recently the Humphrey perimeter has been redesigned with release of the Humphrey field analyser $3^{\text {TM }}$ (Carl Zeiss Meditec Inc, California, USA) series in 2016. The 840-860 models feature a kinetic assessment option that has been remodelled to a userfriendly examiner-operated interface. However, the Humphrey bowl size (i.e., aspheric bowl shape) remains similar to previous models. This is in comparison to the full bowl 
cupola of the Goldmann perimeter, Octopus 900 and other similar perimeters [11].

The aim of this study was to evaluate the diagnostic accuracy of the Humphrey 850 kinetic visual field assessment in comparison to the Octopus 900 to determine the boundary extent of visual fields, uniocular rotations and field of binocular single vision in subjects with normal ocular parameters and visual function.

\section{Methods and materials}

A prospective cross section study was undertaken with institutional ethical approval and in accordance with the Tenets of the Declaration of Helsinki. The study outline is shown in Fig. 1 and consisted of two parts: part 1 for normal visual function measurements and part 2 for comparison to abnormal visual field results.

\section{Subjects}

Subjects were recruited from university and hospital staff. Inclusion criteria were adults aged 18 years or older, sufficient motor ability to sit at the perimeter unaided, able to press the response button, sufficient cognitive ability to understand and follow instructions for performing the test, willingness to undertake testing on both perimeters on the same day, visual acuity of better than $0.3 \log$ MAR in either eye, full ocular motility and absence of manifest strabismus. All subjects underwent perimetry following full explanation of the purpose of the test and procedure and provision of informed consent.

The study protocol consisted of perimetry assessment with both Humphrey and Octopus perimetry on the same day. The order of testing and order of programme were randomised as to which of the two assessment types was used first in order to take fatigue effect in to consideration. A short break of 5-10 min was allowed between testing on either perimeter. Randomisation was undertaken using a computer generated table. Each test included intra-test reliability checks by the addition of repeated vector checks for $20 \%$ of the overall number of vectors tested. The subjects did not undergo a practice of the test prior to the study.

\section{Visual field protocol}

For the purposes of standardisation and comparison in this study, a kinetic protocol was used. Four stimuli of different size and intensity were used (V4e, III4e, I4e and I2e targets). The peripheral visual field boundary was assessed with the V4e, III4e and I4e targets. The blind spot was assessed using a size $\mathrm{I} 4 \mathrm{e}$ target. Central visual field boundary was assessed using a size I2e target. Sixteen vectors were assessed for the peripheral visual field and 12 for the central visual field inclusive of vectors offset from the vertical and horizontal meridia moving centripetally, similar to previously reported testing strategies [7]. Following assessment the response points along each vector were joined to form the isopter for all four targets respectively. Movement of the target was set at $5 \%$ for determination of central and peripheral isopter boundaries and at $3 \%$ for determination of the blind spot boundary. Reliability was determined by manually checking false positive and false negative responses and repeated vector assessments.

\section{Uniocular rotation and field of binocular single vision protocol}

For uniocular cardinal axes, the following were used: (right eye) lateral rectus $0^{\circ}$, superior rectus $67^{\circ}$, inferior oblique $141^{\circ}$, medial rectus $180^{\circ}$, superior oblique $216^{\circ}$ and inferior rectus $293^{\circ}$. The axes for the left eye mirrored those for the right eye. For the binocular field of single vision, the target was moved from central fixation outwards at $30^{\circ}$ degree intervals starting with direct elevation. The head was stabilised with chin and head rests on each perimeter.

The foveal light threshold was established as the smallest, dimmest light visible to each subject and this target was then used to measure the rotations. The use of a foveal light threshold had the advantage of aiding discrimination of the end point of movement in that, when the patient could no longer move his eyes to follow the target, the target moved off the fovea and thus disappeared from the patient's view.

When assessing the range of uniocular ductions, the patient followed the target from central fixation outwards along each axis until the target disappeared from view. When assessing fields of binocular single vision, the patient followed the target from central fixation outwards along each axis until the target disappeared from view or was seen as double at which point the subject pressed the perimeter's response button. The same instructions were provided for both Humphrey and Octopus perimetry. Movement of the target on each perimeter was set at $3 \%$.

\section{Comparison of results}

\section{Data extraction}

The extremity value (outer boundary measurement) was extracted for each vector and recorded as degrees from central fixation. For visual field assessment, four extremity values were taken for direct comparison: nasal $\left(0^{\circ}\right)$, superior $\left(90^{\circ}\right)$, temporal $\left(180^{\circ}\right)$ and inferior $\left(270^{\circ}\right)$. For uniocular rotations, six extremity values were taken according to the six primary action directions of the extraocular muscles. For 
Fig. 1 Study flow chart. $B S V$ binocular single vision, $I I H$ idiopathic intracranial hypertension

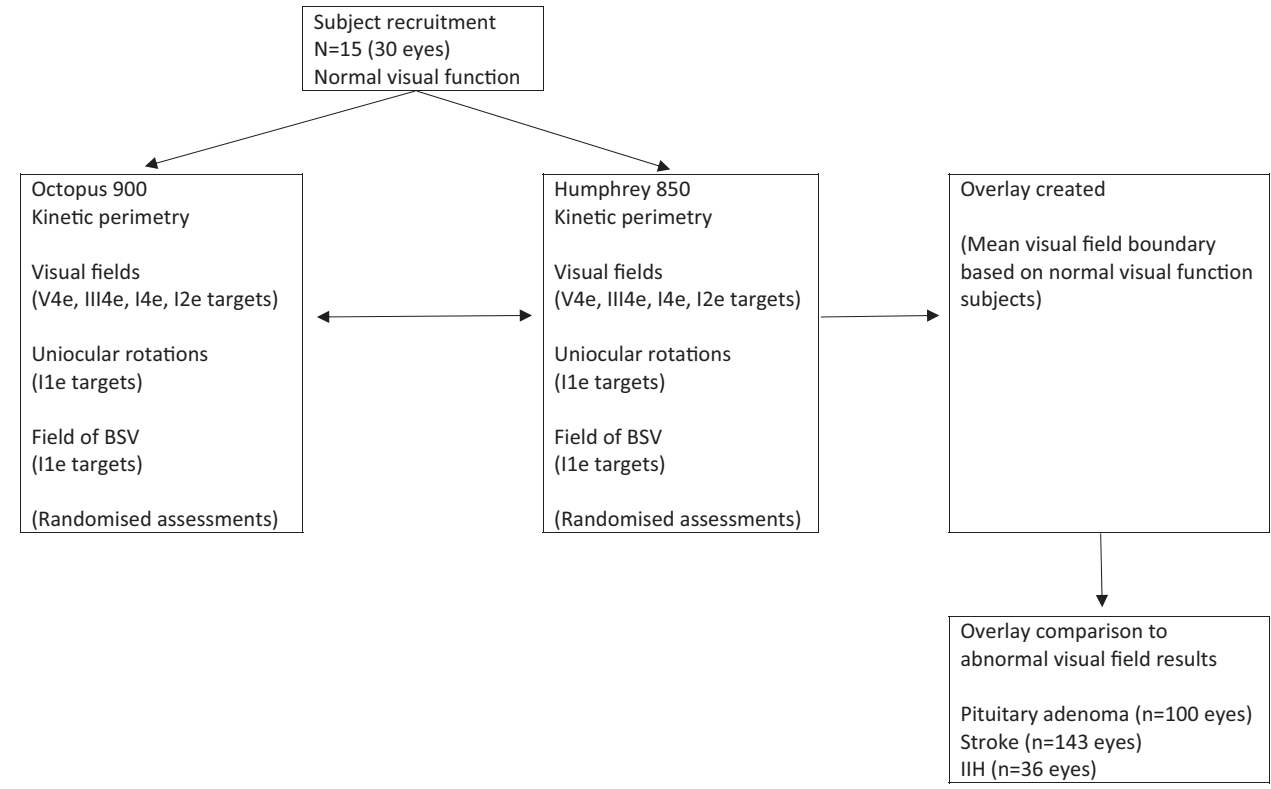

field of binocular single vision, 12 extremity values were taken at 30 degree intervals from 0 to $330^{\circ}$. Duration of test for each test and each eye were captured.

\section{Ocular rotations}

Normative ranges for rotation values were extracted from the published literature for uniocular rotations [10, 12] and fields of binocular single vision [13] for direct comparison of means and standard deviations.

\section{Visual field overlay}

Following extraction of the extremity values for the Humphrey visual field results from part 1 of this study, an overlay was created mapping the maximum peripheral boundaries of the Humphrey visual field. This was overlaid on the visual field results from a databank of recent Octopus 900 kinetic perimetry studies and including chiasmal compression by pituitary adenoma ( $n=50$ patients, 100 eyes), stroke ( $n=72,143$ eyes) and idiopathic intracranial hypertension $(n=18,36$ eyes).

\section{Statistical analysis}

Sample size was based on available subjects that could provide informed consent within the timescale for the study.

Vector extremity values were assessed for goodness of fit (Kolmogorov-Smirnov test) and for skewness. A direct comparison was made for Octopus and Humphrey perimetry results using the statistical package SPSS version 24 (IBM SPSS Statistics, USA). Comparison of vector extremities along with duration of test were compared between perimeters using unpaired $t$ tests.
Descriptive statistics are provided for overlay comparisons where the Octopus kinetic results were the constant factor (known visual field loss). Percentage and number of visual fields captured or not captured are reported.

\section{Results}

\section{Part 1-Normal visual function measurements}

Fifteen subjects (30 eyes) were recruited to this study. Mean age was 38.73 years (SD 9.62) with four males and eleven females. Vector extremity data were not skewed for right versus left eyes (Kolmogorov-Smirnov test) so the data were pooled for analysis for all 30 eyes.

Although intra-test reliability (repeated vectors) measures were built into the testing protocol, values are not reported for these. The Octopus 900 results showed the vector retest positions which could be measured. The Humphrey 850 showed only the second tested position. However, each test was visually monitored by the examiner and it was noted for each test that retest vectors were closely matched to the first response indicating high intra-test reliability across all tests.

\section{Comparison of visual field boundaries}

The mean extremity boundary values for the visual fields are outlined in Fig. 2a-d. A progressive reduction in values was seen for nasal and temporal values from the V4e to the I2e targets (Fig. 2e, f). Significant differences were seen for superior and inferior values for peripheral V4e, III4e and I4e targets reflecting a ceiling effect for superior and inferior 


\begin{tabular}{|c|c|c|c|c|c|}
\hline & \multicolumn{2}{|c|}{ Superior extent $90^{\circ}$} & & \\
\hline \multicolumn{2}{|c|}{ a) V4e target } & H850 & 0900 & & \\
\hline & & $39.47,3.83$ & $53.70,2.91$ & & \\
\hline \multicolumn{2}{|c|}{ Temporal extent $180^{\circ}$} & \multicolumn{2}{|l|}{$P=0.0001$} & \multicolumn{2}{|c|}{ Nasal extent $0^{\circ}$} \\
\hline H850 & 0900 & \multicolumn{2}{|c|}{ Diff: $-14.23,4.58$} & \begin{tabular}{|l|}
$\mathrm{H} 850$ \\
\end{tabular} & 0900 \\
\hline $87.13,2.10$ & $87.03,0.89$ & & & $65.77,13.24$ & $61.20,4.77$ \\
\hline \multicolumn{2}{|l|}{$\mathrm{P}=0.805$} & \multicolumn{2}{|c|}{ Inferior extent $270^{\circ}$} & \multicolumn{2}{|l|}{$P=0.071$} \\
\hline \multicolumn{2}{|c|}{ Diff: $0.1,2.2)$} & $\mathrm{H} 850$ & 0900 & \multicolumn{2}{|c|}{\begin{tabular}{|l|} 
Diff: 4.57. 13.34 \\
\end{tabular}} \\
\hline & & $53.03,2.01$ & $65.63,2.11$ & & \\
\hline & & \multicolumn{2}{|l|}{$P=0.0001$} & & \\
\hline & & \multicolumn{2}{|c|}{ Diff: -12.6, 2.47} & & \\
\hline
\end{tabular}

\begin{tabular}{|c|c|c|c|c|c|}
\hline \multirow{2}{*}{\multicolumn{2}{|c|}{ b) III4e target }} & \multicolumn{2}{|c|}{ Superior extent $90^{\circ}$} & & \\
\hline & & H850 & 0900 & & \\
\hline & & $39.20,4.10$ & $51.77,3.16$ & & \\
\hline \multicolumn{2}{|c|}{ Temporal extent $180^{\circ}$} & \multicolumn{2}{|l|}{$P=0.0001$} & \multicolumn{2}{|c|}{ Nasal extent $0^{\circ}$} \\
\hline H850 & 0900 & \multicolumn{2}{|c|}{ Diff: $-12.57,4.57$} & H850 & 0900 \\
\hline $80.67,7.79$ & $84.83,6.80$ & & & $61.10,10.01$ & $60.33,6.61$ \\
\hline \multicolumn{2}{|l|}{$\mathrm{P}=0.024$} & \multicolumn{2}{|c|}{ Inferior extent $270^{\circ}$} & \multicolumn{2}{|l|}{$P=0.732$} \\
\hline \multicolumn{2}{|c|}{ Diff: $-4.17,9.61$} & H850 & 0900 & \multicolumn{2}{|c|}{ Diff: $0.77,12.15$} \\
\hline & & $52.97,1.83$ & $62.60,2.90$ & & \\
\hline & & \multicolumn{2}{|c|}{$P=0.0001$} & & \\
\hline & & \multicolumn{2}{|c|}{ Diff: -9.63, 2.95} & & \\
\hline
\end{tabular}

\begin{tabular}{|c|c|c|c|c|c|}
\hline & \multicolumn{2}{|c|}{ Superior extent $90^{\circ}$} & & \\
\hline \multicolumn{2}{|c|}{ C) $14 \mathrm{e}$ target } & $\mathrm{H} 850$ & 0900 & & \\
\hline & & $36.97,3.44$ & $44.07,3.59$ & & \\
\hline \multicolumn{2}{|c|}{ Temporal extent $180^{\circ}$} & \multicolumn{2}{|l|}{$P=0.0001$} & \multicolumn{2}{|c|}{ Nasal extent $0^{\circ}$} \\
\hline H850 & 0900 & \multicolumn{2}{|c|}{ Diff: - $7.1,4.44$} & H850 & 0900 \\
\hline $74.77,3.02$ & $74.67,4.25$ & & & $53.57,5.82$ & $51.97,4.61$ \\
\hline \multicolumn{2}{|l|}{$\mathrm{P}=0.836$} & \multicolumn{2}{|c|}{ Inferior extent $270^{\circ}$} & \multicolumn{2}{|l|}{$\mathrm{P}=0.189$} \\
\hline \multicolumn{2}{|c|}{ Diff: $0.1,2.62)$} & H850 & 0900 & \multicolumn{2}{|c|}{ Diff: $1.6,6.52$} \\
\hline & & $50.87,4.26$ & $51.37,6.47$ & & \\
\hline & & \multicolumn{2}{|l|}{$P=0.707$} & & \\
\hline & & \multicolumn{2}{|c|}{ Diff: - $0.5,7.21$} & & \\
\hline
\end{tabular}

\begin{tabular}{|c|c|c|c|c|c|}
\hline \multirow{3}{*}{\multicolumn{2}{|c|}{ d) 12e target }} & \multicolumn{2}{|c|}{ Superior extent $90^{\circ}$} & & \\
\hline & & $\mathrm{H} 850$ & 0900 & & \\
\hline & & $26.87,4.98$ & $20.10,4.77$ & & \\
\hline \multicolumn{2}{|c|}{ Temporal extent $180^{\circ}$} & \multicolumn{2}{|l|}{$P=0.0001$} & \multicolumn{2}{|c|}{ Nasal extent $0^{\circ}$} \\
\hline H850 & 0900 & \multicolumn{2}{|c|}{ Diff: $6.77,5.02$} & H850 & 0900 \\
\hline $51.50,6.76$ & $41.97,8.06$ & & & $33.90,3.85$ & $27.50,4.83$ \\
\hline \multicolumn{2}{|l|}{$P=0.0001$} & \multicolumn{2}{|c|}{ Inferior extent $270^{\circ}$} & \multicolumn{2}{|l|}{$P=0.0001$} \\
\hline \multicolumn{2}{|c|}{ Diff: $9.53,7.08$} & $\mathrm{H} 850$ & 0900 & \multicolumn{2}{|c|}{ Diff: $6.40,4.72$} \\
\hline & & $34.47,4.52$ & $24.43,4.98$ & & \\
\hline & & \multicolumn{2}{|l|}{$\mathrm{P}=0.0001$} & & \\
\hline & & \multicolumn{2}{|c|}{ Diff: $10.03,6.07$} & & \\
\hline
\end{tabular}

e)

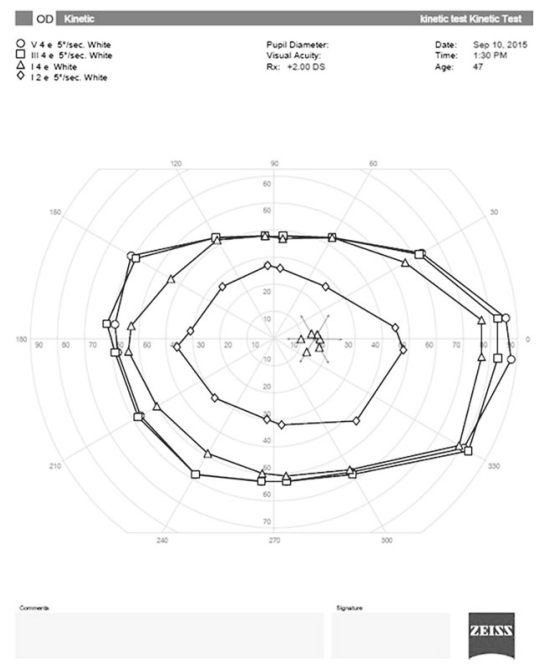

f) Right eye (OD) 10-Sep-2015 17:34:27

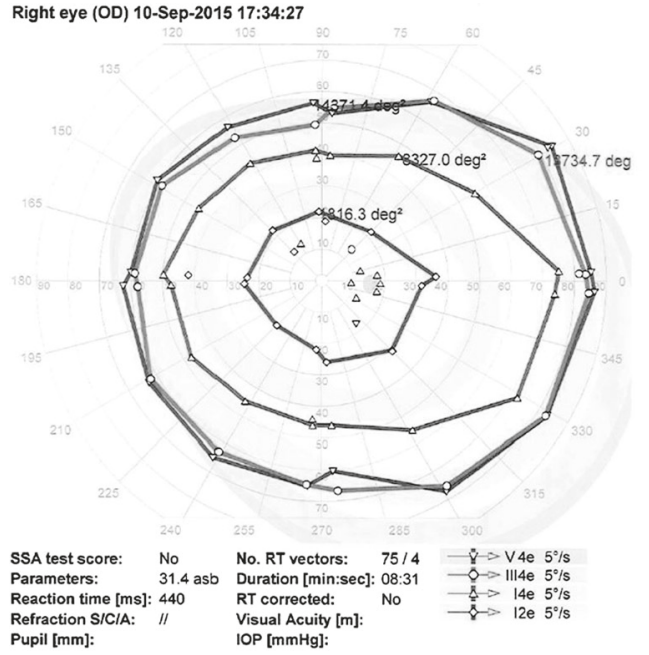

Fig. 2 Kinetic visual field assessment. a Humphrey 850 (b) Octopus 900. Visual field results are displayed for the right eye from the Humphrey 850 (a) and Octopus 900 (b)

boundaries at approximately 40 and 50 degrees respectively $(p=0.0001)$. Mean differences ranging from -14.23 to -7.1 degrees superiorly and -12.6 to -0.5 inferiorly, showed significantly higher Octopus kinetic boundaries than Humphrey in these areas. Conversely, Humphrey visual field boundaries were significantly higher than Octopus for central visual field measured with I2e targets. Duration of visual field testing was significantly longer for Octopus perimetry (mean difference of $2.45 \mathrm{~min}$ ) reflecting the extra time taken to measure the larger visual field obtained with the Octopus 900.

\section{Comparison of field of binocular single vision}

Mean extremity values for fields of binocular single vision are shown in Fig. 3a. Significant differences were found for all three superior extremities $(60,90$ and 120 degree vectors) ranging from -4.47 to -5.87 , and for two inferior extremities (240 and 300 degree vectors) ranging from -4.87 to -6.13 ; Octopus 900 measured larger extremities in these vector positions (Fig. 3b, c). In addition, for the three superior extremities on Humphrey perimetry, no responses were recorded for $6(40 \%)$ subjects at $60^{\circ}$, for 8 $(53.3 \%)$ subjects at $90^{\circ}$ and for $5(33.3 \%)$ subjects at $120^{\circ}$. At inferior extremities, no responses were recorded for 6 $(40 \%)$ subjects at $270^{\circ}$ and for $1(6.6 \%)$ subject at $300^{\circ}$. There were no significant differences between perimeters for nasal and temporal vectors. Duration of test was not significantly different between perimeters.

\section{Comparison of uniocular rotations}

The mean extremity values for uniocular rotations are outlined in Fig. 4a. No significant differences were found for 
a

\begin{tabular}{|c|c|c|c|c|c|c|c|c|c|}
\hline & \multicolumn{2}{|c|}{ Sup-left extent $120^{\circ}$} & \multicolumn{2}{|c|}{ Superior extent $90^{\circ}$} & \multicolumn{2}{|c|}{ Sup-right extent $60^{\circ}$} & & \\
\hline \multirow{2}{*}{\multicolumn{2}{|c|}{ I1e target }} & \multirow{2}{*}{\begin{tabular}{|l|} 
H850 \\
$38.40,8.02$ \\
\end{tabular}} & \multirow{2}{*}{$\begin{array}{l}0900 \\
44.27,8.12 \\
\end{array}$} & \begin{tabular}{|l|l} 
H850 & 0900 \\
\end{tabular} & & & & & \\
\hline & & & & $34.20,9.81$ & $40.93,7.08$ & $38.33,8.01$ & $42.80,8.33$ & & \\
\hline \multicolumn{2}{|c|}{ Sup-left extent $150^{\circ}$} & \multicolumn{2}{|l|}{$\mathrm{P}=0.01$} & \multicolumn{2}{|l|}{$\mathrm{P}=0.005$} & \multicolumn{2}{|l|}{$\mathrm{P}=0.012$} & \multicolumn{2}{|c|}{ Sup-right extent $30^{\circ}$} \\
\hline \begin{tabular}{|l|}
$\mathrm{H} 850$ \\
\end{tabular} & 0900 & \multicolumn{2}{|c|}{ Diff: $-5.87,7.63$} & \multicolumn{2}{|c|}{ Diff: $-6.73,7.88$} & \multicolumn{2}{|c|}{ Diff: $-4.47,6.01$} & \begin{tabular}{|l|}
$\mathrm{H} 850$ \\
\end{tabular} & 0900 \\
\hline \begin{tabular}{|l|}
$45.53,8.44$ \\
\end{tabular} & $47.87,7.58$ & & & & & & & $46.67,12.28$ & $46.93,9.85$ \\
\hline \multicolumn{2}{|l|}{$\mathrm{P}=0.198$} & & & & & & & \multicolumn{2}{|l|}{$\mathrm{P}=0.874$} \\
\hline \multicolumn{2}{|c|}{ Diff: -2.33, 6.68} & & & & & & & \multicolumn{2}{|c|}{ Diff: $-0.27,6.41$} \\
\hline \multicolumn{2}{|c|}{ Leftward extent $180^{\circ}$} & & & & & & & \multicolumn{2}{|c|}{ Rightward extent $0^{\circ}$} \\
\hline \begin{tabular}{|l|}
$\mathrm{H} 850$ \\
\end{tabular} & 0900 & & & & & & & \begin{tabular}{|l|}
$\mathrm{H} 850$ \\
\end{tabular} & 0900 \\
\hline $44.80,9.51$ & $48.20,7.26$ & & & & & & & $46.87,11.27$ & $46.67,7.92$ \\
\hline \multicolumn{2}{|l|}{$P=0.106$} & & & & & & & \multicolumn{2}{|l|}{$P=0.941$} \\
\hline \multicolumn{2}{|c|}{\begin{tabular}{|l|} 
Diff: $-3.14,7.6$ \\
\end{tabular}} & & & & & & & \multicolumn{2}{|c|}{\begin{tabular}{|l|} 
Diff: $0.2,10.28$ \\
\end{tabular}} \\
\hline \multicolumn{2}{|c|}{ Inf-left extent $210^{\circ}$} & & & & & & & \multicolumn{2}{|c|}{ Inf-right extent $330^{\circ}$} \\
\hline \begin{tabular}{|l|}
$\mathrm{H} 850$ \\
\end{tabular} & 0900 & & & & & & & \begin{tabular}{|l|}
$\mathrm{H} 850$ \\
\end{tabular} & 0900 \\
\hline \begin{tabular}{|l|}
$41.33,8.32$ \\
\end{tabular} & $46.40,9.89$ & & & & & & & $43.73,8.22$ & $45.47,9.91$ \\
\hline \multicolumn{2}{|l|}{$\mathrm{P}=0.057$} & \multicolumn{2}{|c|}{ Inf-left extent $240^{\circ}$} & \multicolumn{2}{|c|}{ Inferior extent $270^{\circ}$} & \multicolumn{2}{|c|}{ Inf-right extent $300^{\circ}$} & $\mathrm{P}=0.483$ & \\
\hline \begin{tabular}{|l|} 
Diff: -5.07, \\
\end{tabular} & & $\mathrm{H} 850$ & 0900 & H850 & 0900 & $\mathrm{H} 850$ & 0900 & Diff: $-1.73,9$ & \\
\hline & & $45.47,7.99$ & $50.33,9.33$ & $53.13,8.51$ & $52.73,7.30$ & $44.93,7.45$ & $51.07,10.82$ & & \\
\hline & & $P=0.5$ & & $\mathrm{P}=0.881$ & & $P=0.02$ & & & \\
\hline & & Diff: $-4.87,8$ & & Diff: $0.4,10$. & & Diff: $-6.13,9$ & & & \\
\hline
\end{tabular}

Means, SD standard deviation, $p$ values of paired samples $t$ test

Difference in means

b

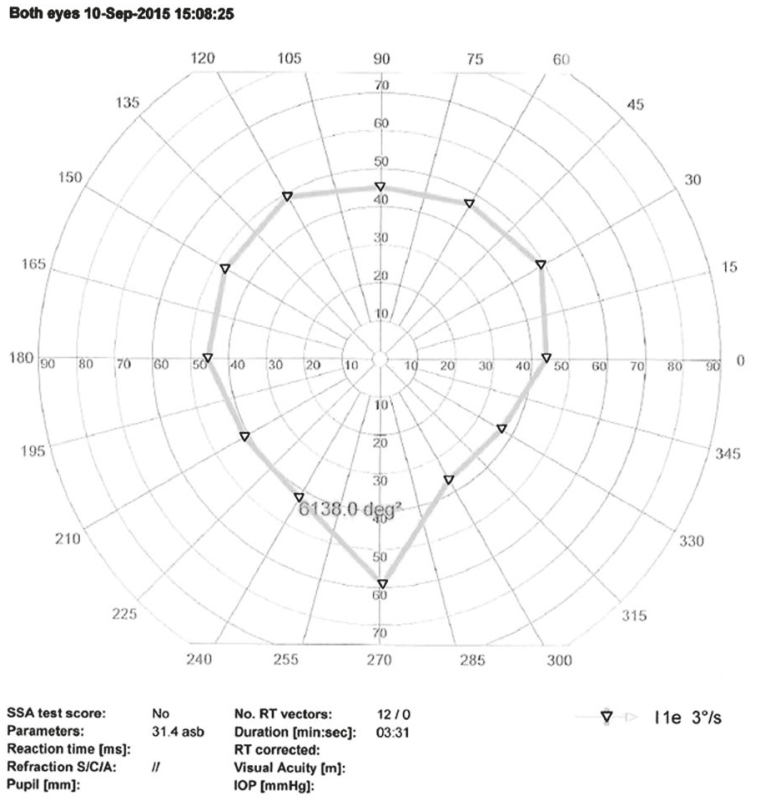

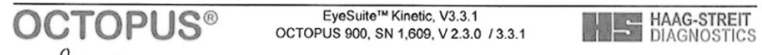
Retost

Fig. 3 Field of binocular single vision. a Humphrey 850 b Octopus 900. The fields of binocular single vision are displayed from the Humphrey 850 (a) and Octopus 900 (b)

these mean values between perimeters. However, ceiling effects were found for 8 subjects (Fig. 4b, c). For superior rectus values, no responses were recorded on Humphrey perimetry for 12 eyes (40\%), for inferior rectus 14 eyes
C
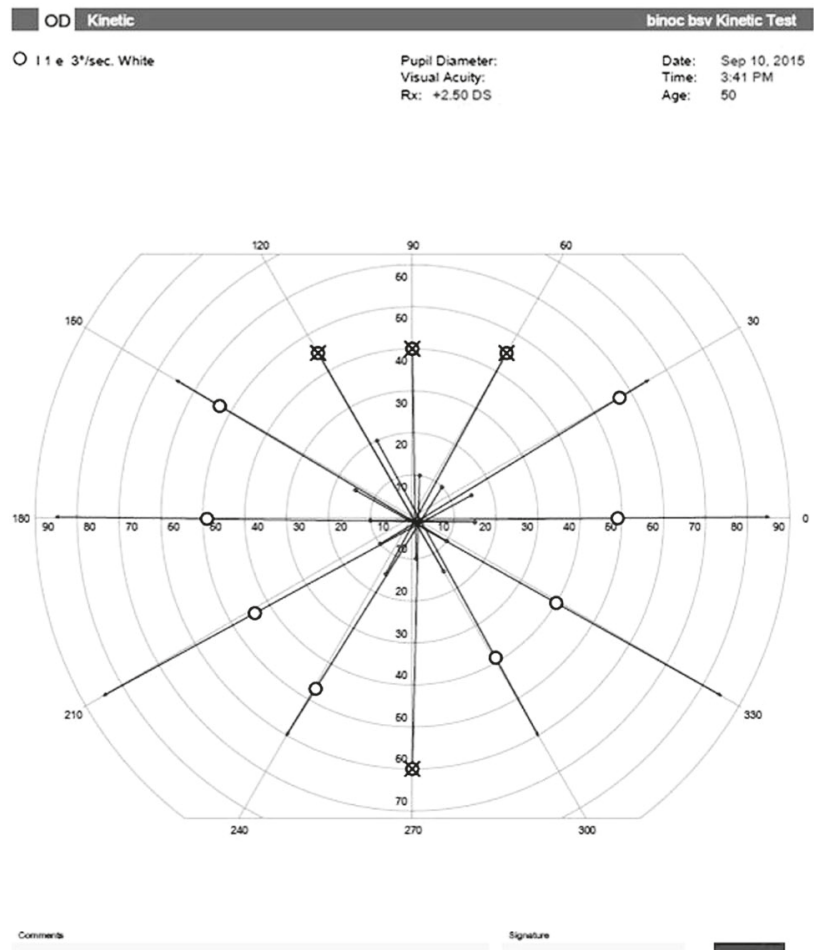

ABISS
(46.6\%) and for inferior oblique 2 eyes (6.6\%). Octopus measurements were higher for each of these subjects for these respective vectors. Duration of test was not significantly different between perimeters. 


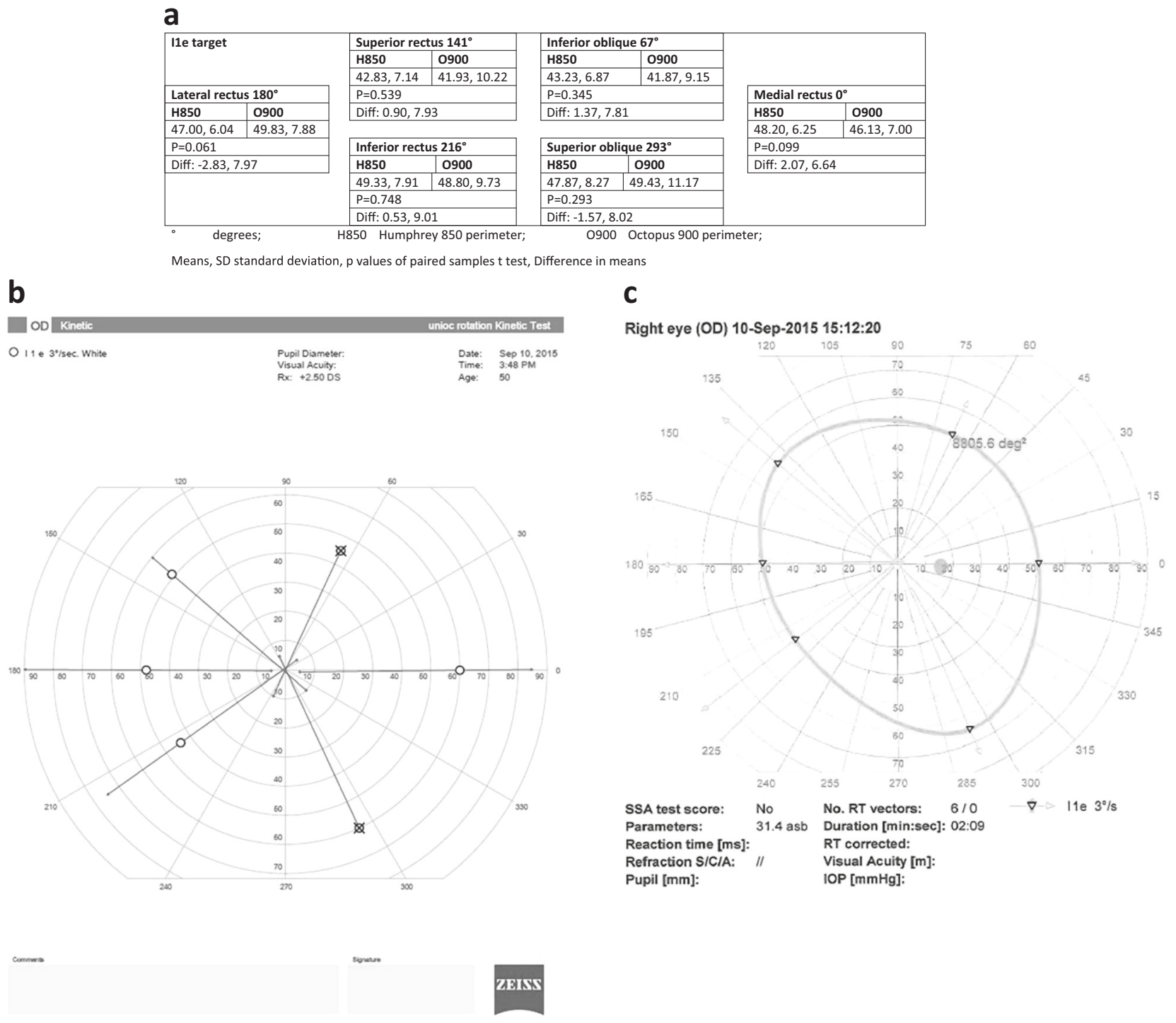

Fig. 4 Uniocular rotations. a Humphrey 850 (b) Octopus 900. Uniocular rotation results are displayed for the right eye from the Humphrey 850 (a) and Octopus 900 (b)

\section{Part 2-Abnormal visual field results}

A Humphrey 850 kinetic visual field overlay was placed over the Octopus kinetic visual field results from 140 patients (279 eyes) with known abnormal visual fields due to diagnoses of chiasmal compression (100 eyes), stroke (143 eyes) or idiopathic intracranial hypertension (36 eyes). Comparisons were made for I4e and I2e targets. Overall, visual field loss present on the Octopus 900 result was detected within the Humphrey kinetic overlay in 255 eyes (91.4\%) when using the I4e target. Visual field loss was detected in 265 eyes $(95 \%)$ when using the I2e target. All stroke-induced homonymous hemianopia defects and all idiopathic intracranial hypertension visual field defects were detected using the overlay. Missed visual field defects (24
(8.6\%) missed with I4e target and 14 (5\%) missed with I2e target) all included superior peripheral defects in cases of chiasmal compression and stroke (Fig. 5).

\section{Part 3-Ocular rotation comparisons}

For fields of binocular single vision, in comparison to published normative ranges, range of movement was considerably reduced for Humphrey 850 rotations in elevated positions from 30 through to 150 degrees (Table 1) with differences of up to 14 degrees in direct elevation positions.

For uniocular rotations, differences to published normative ranges were less for the Humphrey 850 values. The greatest difference was for the inferior rectus vector with a difference of about 12 degrees (Table 1). 
OS 08-Nov-2009 10:25:50

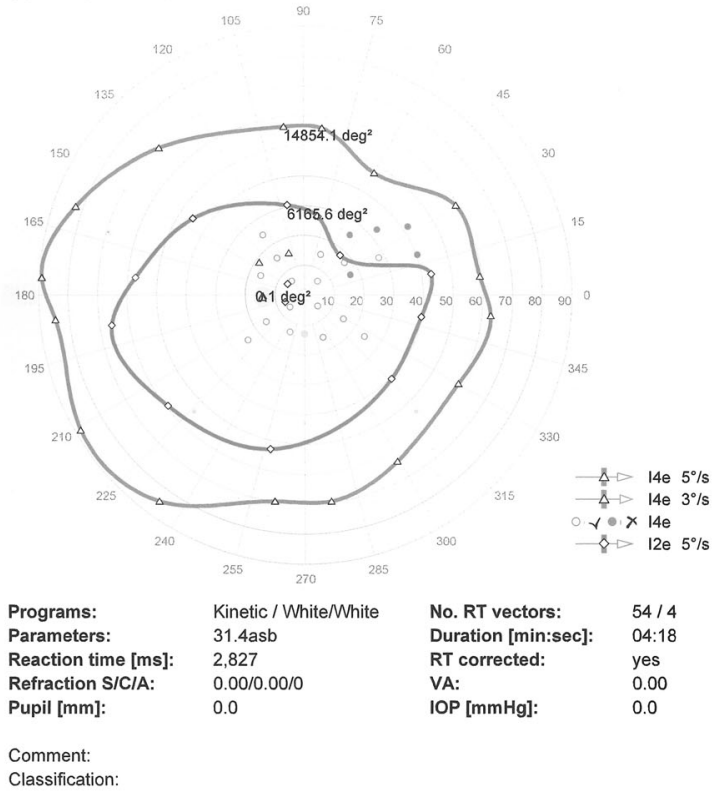

OD 08-Nov-2009 10:13:54

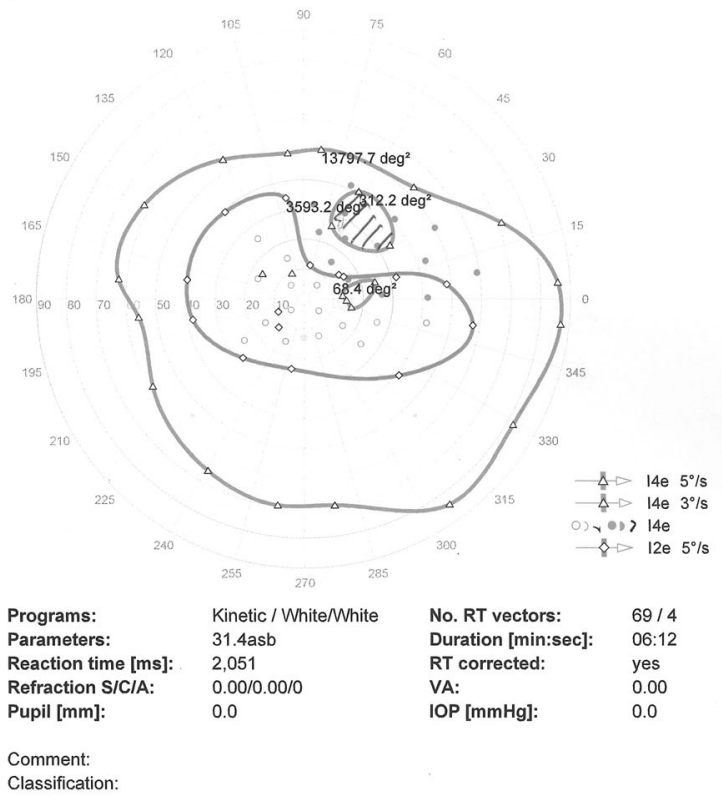

OCTOPUS ${ }^{\circledR}$ EyeSuiter" Kinetic $V 2.0 .4$

OCTOPUS 900 , SNr. 328 v9.99

Fig. 5 Octopus 900 visual field result—defect not captured by Humphrey overlay. Partial right-sided superior homonymous quadrantanopia caused by stroke. Detection of the defect failed with I4e target of Humphrey 850 overlay but with detection possible using the I2e target

Table 1 Ocular rotations

\begin{tabular}{|c|c|c|c|c|c|c|c|c|c|c|c|c|}
\hline \multicolumn{13}{|c|}{ A Comparison of fields of binocular single vision on every $30^{\circ}$ meridian } \\
\hline Meridian & $0^{\circ}$ & $30^{\circ}$ & $60^{\circ}$ & $90^{\circ}$ & $120^{\circ}$ & $150^{\circ}$ & $180^{\circ}$ & $210^{\circ}$ & $240^{\circ}$ & $270^{\circ}$ & $300^{\circ}$ & $330^{\circ}$ \\
\hline \multicolumn{13}{|l|}{ Yagasaki et al. [13] } \\
\hline Mean & 48. & 52.2 & 53.1 & 55.9 & 53.1 & 51.3 & 47.5 & 49.3 & 49.1 & 49.4 & 48.6 & 48.3 \\
\hline SD & 5.2 & 4.8 & 4.9 & 4.5 & 4.0 & 6.8 & 6.0 & 6.6 & 5.7 & 5.3 & 6.3 & 5.9 \\
\hline \multicolumn{13}{|l|}{ Current study } \\
\hline Mean & 46.8 & 46.67 & 38.33 & 34.20 & 38.40 & 45.53 & 44.80 & 41.33 & 45.47 & 53.13 & 44.93 & 43.73 \\
\hline SD & 11.2 & 12.28 & 8.01 & 9.81 & 8.02 & 8.44 & 9.51 & 8.32 & 7.99 & 8.51 & 7.45 & 8.22 \\
\hline \multicolumn{13}{|c|}{ B Comparison of extraocular muscle rotation measurements } \\
\hline & \multicolumn{2}{|c|}{ Superior oblique } & \multicolumn{2}{|c|}{ Inferior rectus } & \multicolumn{2}{|c|}{ Inferior oblique } & \multicolumn{2}{|c|}{ Superior rectus } & \multicolumn{2}{|c|}{ Lateral rectus } & \multicolumn{2}{|c|}{ Medial rectus } \\
\hline & Mean & & Mean & & Mean & & Mean & & Mean & & Mean & \\
\hline Haggerty et al. [12] & 48.5 & $41.8-55.2 \mathrm{CI}$ & 61.9 & 54.4-69.4 CI & 45.7 & 39.1-52.3 CI & 42.7 & $36.1-49.3 \mathrm{CI}$ & 52.1 & $44.7-59.5 \mathrm{CI}$ & 50.6 & $45.2-56.1 \mathrm{Cl}$ \\
\hline Hanif et al. [10] & 49.45 & $6.65 \mathrm{SD}$ & 61.15 & $8.71 \mathrm{SD}$ & 44.25 & $10.27 \mathrm{SD}$ & 42.5 & $9.64 \mathrm{SD}$ & 51.7 & 7.97SD & 49.25 & $7.94 \mathrm{SD}$ \\
\hline Current study & 47.87 & 8.27SD & 49.33 & 7.91SD & 43.23 & $6.87 \mathrm{SD}$ & 42.83 & 7.14SD & 47.00 & $6.04 \mathrm{SD}$ & 48.20 & $6.25 \mathrm{SD}$ \\
\hline
\end{tabular}

Mean values relate to vector rotations in degrees. SD represent standard deviations and CI represent $95 \%$ confidence intervals for mean values

\section{Discussion}

Across all assessments of visual fields, uniocular rotations and fields of binocular single vision, the Humphrey 850 showed ceiling effects for measurements in the superior and inferior visual fields, at approximately 40 degrees superiorly and 50 degrees inferiorly. This corresponds to the dimensions of the aspheric bowl such that stimuli do not extend beyond these limits.
For visual field assessment, the average superior boundary with an III4e target was $51.77^{\circ}$ for Octopus 900 and $39.20^{\circ}$ for Humphrey 850 with a mean difference of $-12.57^{\circ}$. The average inferior boundary was $62.6^{\circ}$ for Octopus 900 and $52.97^{\circ}$ for Humphrey 850 with a mean difference of $-9.63^{\circ}$. Similar significant differences were also found with $\mathrm{V} 4 \mathrm{e}$ and I4e targets. Ceiling effects were not found for I2e targets when, conversely, a larger central visual field was measured by the Humphrey 850 . For this latter discrepancy in values, 
differences must be considered in relation to background illumination, size of stimulus and stimulus intensity. Slight variances across perimeters will show greatest effects with the dimmest stimuli as contrast variances are smallest. We postulate that such variance explains the I2e stimuli differences. Variances of $+25 /-20 \%$ are allowable within normal ranges for perimeters for background light intensity, $+20 /-15 \%$ for stimulus size and $+25 /-2 \%$ for contrast, according to EU guidelines [14].

Kinetic visual field assessment is typically indicated for neuro-ophthalmic conditions, for blepharoptosis assessment, in people with poor central fixation or visual acuity, in those with poor cognition, those who are unwell, for children and those who otherwise struggle with static threshold perimetry $[4-10,15,16]$. Previous comparisons of full bowl kinetic perimeters have shown reproducible findings [17-19]. Many conditions causing both central and peripheral visual field loss are likely to impact the visual field falling within the mapped boundaries of the Humphrey 850 , even when accounting for the superior and inferior ceiling effects. Our analysis of common neuro-ophthalmic conditions using a Humphrey 850 boundary overlay show this to be the case with diagnostic accuracy achieved for stroke-induced homonymous hemianopia and for idiopathic intracranial hypertension with constricted visual fields and enlarged blind spots. The consistent exception, however, was for peripheral superior visual field defects (e.g., chiasmal compressions by pituitary tumours). Our results showed that the Humphrey 850 missed $8.6 \%$ of such defects when using the $\mathrm{I} 4 \mathrm{e}$ target (the smallest target used for delineating the peripheral kinetic visual field boundary), outside the ceiling of 40 degrees superiorly. This finding is similar to our previous comparison of Octopus kinetic perimetry and Humphrey full field 120 static testing in which superior peripheral visual field defects were not detected [20]. Again, this was due to the constraints of the aspheric bowl limiting superior visual field static testing. To avoid the limits imposed by aspheric perimetry bowl designs, we recommend full bowl kinetic perimetry to facilitate exploration of superior and inferior visual field extremities. In the example of chiasmal pathology, this is likely to enhance diagnostic accuracy.

Previous studies of uniocular rotations and fields of binocular single vision report the benefit of plotting extraocular muscle restrictions and extent of maintenance of single vision as part of diagnostic accuracy and subsequently in the monitoring of improvement, deterioration or stability of the condition [12, 21, 22]. Measurements of the field of binocular single vision showed significant differences in mean rotations in the superior and inferior gaze positions. Furthermore, a number of vectors had a 'null' response. Null response indicated that the subject could still see and follow the target when it disappeared because it had reached the limit of the
Humphrey bowl constraints. Comparisons of Humphrey 850 results to normative values published in the literature $[10,12,13]$ reinforced the differences found for superior and inferior gaze positions. This indicates a potential limitation of the Humphrey 850 in fully evaluating the degree of rotation in these particular positions of gaze. Although the horizontal measurements were not affected when using the Humphrey 850 , we suggest the ceiling effects found for superior and inferior rotations are likely to hamper reliable and accurate measurements relating to conditions affecting the superior and/or inferior rectus and oblique extraocular muscles [23-25] as, for these related rotation measures, the stimulus disappears whilst the patient may still be able to see and track the stimulus further than the perimeter allows.

\section{Limitations}

Our study had a small sample size of fifteen subjects. However, assessments followed a robust standardised and repeatable testing protocol in which reliable visual field and ocular rotation measurements were obtained. A further limitation was our use of a Humphrey 850 boundary overlay and comparisons to published normative ranges. We chose this option to minimise assessment burden to patients for this initial exploration of the Humphrey 850 kinetic capabilities. We compensated by using the overlay on 140 patient results (279 eyes) across a variety of common neuro-ophthalmic conditions. However, we acknowledge further study of clinical populations with comparison of Humphrey 850 versus full bowl kinetic perimetry (e.g., Octopus 900) is warranted to confirm our findings in relation to restrictive effects of superior and inferior celling effects due to an aspheric bowl.

\section{Conclusions}

The Humphrey 850 kinetic programme is a much improved user-friendly operating interface than previous Humphrey perimeters, which enables assessment of visual fields, uniocular rotations and field of binocular single vision. Its disadvantage over traditional kinetic perimetry is the ceiling effects in the superior (approximate 40 degree cap) and inferior (approximate 50 degree cap) visual field, caused by restrictions of its aspheric bowl. This may impair its diagnostic accuracy for fields of binocular single vision and uniocular rotations where ocular motility conditions require accurate measurements in superior and/or inferior gaze positions. Whilst diagnostic accuracy for visual field assessment is high for many neuro-ophthalmic conditions in which the visual field loss extends to the central visual field and/or along horizontal meridians, there are potential diagnostic accuracy issues for conditions that specifically 
impact the peripheral superior and/or inferior visual field first with the most common example being chiasmal compression. Further study is warranted with such specific patient populations.

\section{Summary}

\section{What was known before}

- Static and kinetic perimetry options are commonly used in the diagnosis and monitoring of ocular disease.

\section{What this study adds}

- Humphrey kinetic perimetry is a useful option but may underestimate or miss visual field loss in peripheral superior/inferior visual field due to ceiling effects

Author contributions FR provided oversight for the study and led the writing of the paper. FR, $\mathrm{LH}, \mathrm{KH}, \mathrm{MM}, \mathrm{CN}$ contributed to data collection, reviewing the draft paper and approving the final version.

\section{Compliance with ethical standards}

Conflict of interest The authors do not have any commercial or proprietary interest in the Octopus 900 perimeter and Humphrey field analyzer or Haag Streit International and Carl Zeiss Meditec. Haag Streit provided the loan of the Octopus 900 perimeter for the conduct of this research study. Carl Zeiss Meditec provided the loan of the Humphrey 850 perimeter for the conduct of this research study. Haag Streit and Carl Zeiss Meditec had no role in the design or conduct of this research.

Publisher's note: Springer Nature remains neutral with regard to jurisdictional claims in published maps and institutional affiliations.

\section{References}

1. Johnson CA, Nelson-Quigg JM. A prospective 3-year study of response properties of normal subjects and patients during automated perimetry. Ophthalmology. 1993;100:269-74.

2. Heijl A, Patella VM. The field analyzer primer. Essenital perimetry. 3rd ed. California: Carl Zeiss Meditec; 2002.

3. Wild JM, Pacey IE, Hancock S, Cunliffe IA. Between-algorithm, between-individual differences in normal perimetric sensitivity: Full threshold, FASTPAC and SITA. Swedish Interactive Threshold Algorithm. Invest Ophthalmol Vis Sci. 1999;40:1152-61.

4. Cummings MF, van Hof-van Duin J, Mayer DL, Hansen RM, Fulton AB. Visual fields of young children. Behav Brain Res. 1988;29:7-16.

5. Lepore FE. The preserved temporal crescent: The clinical implications of an 'endangered' finding. Neurology. 2001;57:1918-21.
6. Blamires TL, Reeves BC. Vision defects in patients with perichiasmal lesions. Optom Vis Sci. 1996;73:572-8.

7. Rowe FJ, Sarkies NJ. Assessment of visual function in idiopathic intracranial hypertension: a prospective study. Eye. 1998;12:111-8.

8. Agrawal S, Mayer DL, Hansen RM, Fulton AB. Visual fields in young children treated with Vigabatrin. Optom Vis Sci. 2009;86:767-73.

9. Donahue SP. Perimetry techniques in neuro-ophthalmology. Curr Opin Ophthalmol. 1999;10:420-8.

10. Rowe FJ, Hanif S. Uniocular and binocular fields of rotation measures: Octopus versus Goldmann. Graefes Arch Clin Exp Ophthalmol. 2011;249:909-19.

11. Hashimoto S, Matsumoto C, Eura M, Okuyama S, Shimomura Y. Evaluation of kinetic programs in various automated perimeters. Jpn J Ophthalmol. 2017;61:299-306.

12. Haggerty H, Richardson S, Mitchell KW, Dickinson AJ. A modified method for measuring uniocular fields of fixation. Arch Ophthalmol. 2005;123:356-62.

13. Yagasaki T, Oya Y, Maeda M, Tsukui M. Modified method for assessment of the binocular fusional field in patients with suppression. Jpn J Ophthalmol. 2009;53:138-44.

14. ISO: International Standard. Ophthalmic instruments-perimeters. Geneva, Switzerland: ISO; 1999.

15. Dersu I, Wiggins MN, Luther A, Harper R, Chacko J. Understanding visual fields, Part I; Goldmann perimetry. J Ophthalmic Med Technol. 2006;2:1-10.

16. Patel D, Cumberland P, Russell-Eggitt I, Walters B, Rahi J, OPTIC study group. How should we assess reliability of visual field assessment in children?. Invest Ophthalmol Vis Sci. 2013;54:5675.

17. Ramirez AM, Chaya CJ, Gordon LK, Giaconi JA. A comparison of semiautomated versus manual Goldmann kinetic perimetry in patients with visually significant glaucoma. J Glaucoma. 2008;17:111-7.

18. Pineles SL, Volpe NJ, Miller-Ellis E, Galetta SL, Sankar PS, Shindler KS, et al. Automated combined kinetic and static perimetry: an alternative to standard perimetry in patients with neuroophthalmic disease and glaucoma. Arch Ophthalmol. 2006;124:363-9.

19. Rowe FJ, Rowlands A. Comparison of diagnostic accuracy between Octopus 900 and Goldmann kinetic visual fields. BioMed Res Int. 2014;2014:214829.

20. Rowe FJ, Noonan CP, Manuel M. Comparison of Octopus semiautomated kinetic perimetry and Humphrey peripheral static perimetry in neuro-ophthalmic cases. ISRN Ophthalmol. 2013;2013:753202.

21. Rowe FJ, Chenye CP, Garcia-Fiñana M, Noonan C, Howard C, Smith J, et al. Detection of visual field loss in pituatary disease: peripheral kinetic versus central static. Neuro-Ophthalmol. 2015;39:116-24.

22. Hanif S, Rowe FJ, O'Connor A. The comparative analysis of assessment methods for uniocular fields lof fixation. Br Ir Orthopt J. 2009;6:47-51.

23. Roper-Hall G. Duction measurements in limited rotations. Br Ir Orthopt J. 1975;32:72-6.

24. Esser J, Melzer I. Comparison of monocular excursion measurements in normals and in patients with motility disorders. Rome, Italy: International Strabismological Association; 1986. p. 285-92.

25. Steel DHW, Hoh HB, Potts MJ, Harrad RA. Uniocular fields of fixation in thyroid eye disease. Eye. 1995;9:348-51. 